\title{
Slow-light switching in nonlinear Bragg-grating coupler
}

\author{
Sangwoo Ha, Andrey A. Sukhorukov, and Yuri S. Kivshar \\ Nonlinear Physics Centre and Centre for Ultra-high bandwidth Devices for Optical Systems (CUDOS), \\ Research School of Physical Sciences and Engineering, \\ Australian National University, Canberra, ACT 0200, Australia
}

\begin{abstract}
We study propagation and switching of slow-light pulses in nonlinear couplers with phase-shifted Bragg gratings. We demonstrate that power-controlled nonlinear self-action of light can be used to compensate dispersion-induced broadening of pulses through the formation of gap solitons, to control pulse switching in the coupler, and to tune the propagation velocity.
\end{abstract}

(C) 2018 Optical Society of America

OCIS codes: $190.5530,230.4320$

It is known that the speed of light can be dramatically reduced in photonic-crystal waveguides with a periodic modulation of the optical refractive index ${ }^{1,2,3}$. In the regime of slow light the photon-matter interactions are dramatically enhanced ${ }^{4}$ allowing for all-optical control and manipulation. In particular, nonlinear self-action can be used to dynamically tune the velocity of pulses propagating in nonlinear Bragg gratings ${ }^{5}$. At the same time, nonlinearity can support pulse self-trapping in the form of a gap soliton which profile remains undistorted during propagation ${ }^{\underline{5}}$, overcoming the limitations of linear devices due to dispersion-induced pulse broadening 6 .

Nonlinear effects can also enable ultra-fast all-optical pulse switching. Pulse routing between output ports controlled by optical power was demonstrated in directional couplers $7.8,9$. Additional flexibility in mode conversion with applications to add-drop filters $10,11,12$ is realized by combining directional coupler geometry and Bragg gratings in optical fibers $\frac{13}{}$ or planar photonic structures ${ }^{14}$ which operation can be tuned all-optically 15 , and gap solitons can also exist in the nonlinear regime $16,17,18$.

In this Letter, we suggest novel possibilities for dynamic manipulation of slow-light pulses which frequency is tuned in the vicinity of Bragg resonance. The pulse dynamics can be modeled by a set of coupled-mode nonlinear equations $\frac{19}{19}$ for the normalized slowly varying envelopes of the forward $\left(u_{n}\right)$ and backward $\left(w_{n}\right)$ propagating fields in each of waveguides $n=1,2$, $-i \partial u_{n} / \partial t=i \partial u_{n} / \partial z+C u_{3-n}+\rho_{n} w_{n}+\gamma\left(\left|u_{n}\right|^{2}+\right.$ $\left.2\left|w_{n}\right|^{2}\right) u_{n},-i \partial w_{n} / \partial t=-i \partial w_{n} / \partial z+C w_{3-n}+\rho_{n}^{*} u_{n}+$ $\gamma\left(\left|w_{n}\right|^{2}+2\left|u_{n}\right|^{2}\right) w_{n}$, where $t$ and $z$ are the dimensionless time and propagation distance normalized to $t_{s}$ and $z_{s}$, respectively, $C$ is the coupling coefficient for the modes of the neighboring waveguides, $\rho_{n}$ characterizes the amplitude and phase of the Bragg gratings, $\gamma$ is the nonlinear coefficient, and the group velocity far from the Bragg resonance is normalized to unity. The scaling coefficients are $t_{s}=\lambda_{0}^{2}\left|\rho_{1}\right| /\left(\pi c \Delta \lambda_{0}\right)$ and $z_{s}=t_{s} c / n_{0}$, where $c$ is the speed of light in vacuum, $\lambda_{0}$ is the wavelength in vacuum, $\Delta \lambda_{0}$ is the width of Bragg resonance for an individual waveguide, $n_{0}$ is the effective refractive index in the absence of a grating. To be specific, in numerical ex-
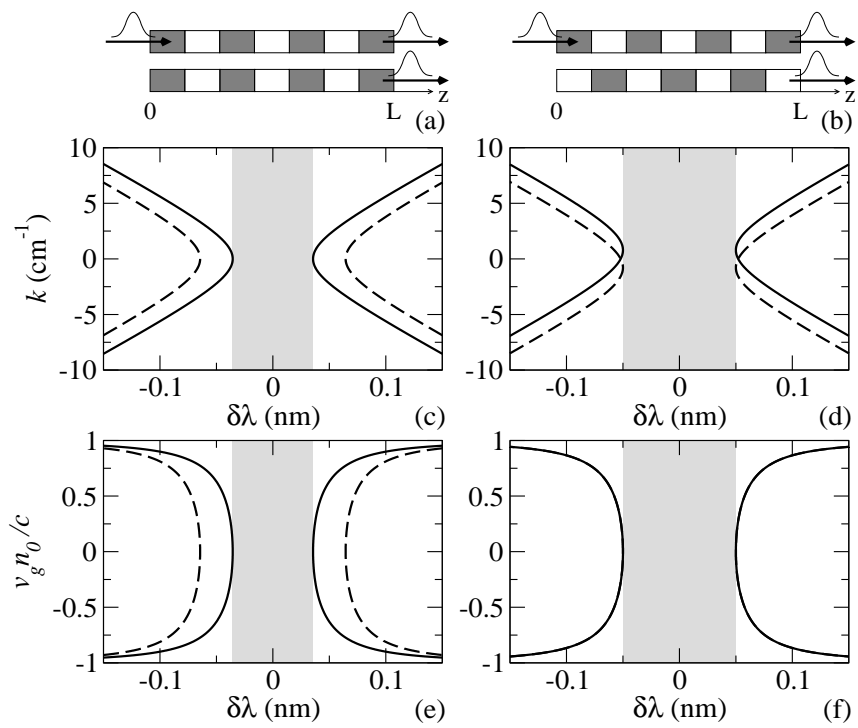

Fig. 1. (a,b) Schematic of directional couplers with (a) in-phase $\left(\rho_{1}=\rho_{2}=0.5\right)$ or (b) out-of-phase $\left(\rho_{1}=\right.$ $\left.-\rho_{2}=0.5\right)$ Bragg gratings. (c,d) Characteristic dispersion and (e,f) normalized group velocity dependence on wavelength detuning for the case of in-phase $(\mathrm{c}, \mathrm{e})$ and out-of-phase (d,f) gratings. For all the plots $C \simeq 0.144$.

amples we set $\gamma=10^{-2}, \lambda_{0}=1550.63 \mathrm{~nm}, \Delta \lambda_{0}=0.1 \mathrm{~nm}$, $t_{s} \simeq 12.8 \mathrm{ps}, z_{s} \simeq 1.8 \mathrm{~mm}$ corresponding to characteristic parameters of fiber Bragg gratings 5.13 .

We consider the case of identical waveguides and analyze the effect of a phase shift $(\varphi)$ between the otherwise equivalent waveguide gratings with $\rho_{1}=\rho$ and $\rho_{2}=\rho \exp (i \varphi)$ (with no loss of generality, we take $\rho$ to be real and positive), see schematic illustrations in Figs. 1 $(a, b)$. It was shown that the grating shift can strongly modify the reflectivity of modes with different symmetries $11,13,14$, and we investigate how this structural parameter affects the properties of slow-light pulses.

In the linear regime, wave propagation is fully defined through the Floquet-Bloch eigenmode solutions of the form, $u_{n}=U_{n} \exp (i \beta z-i \omega t), w_{n}=W_{n} \exp (i \beta z-i \omega t)$. 

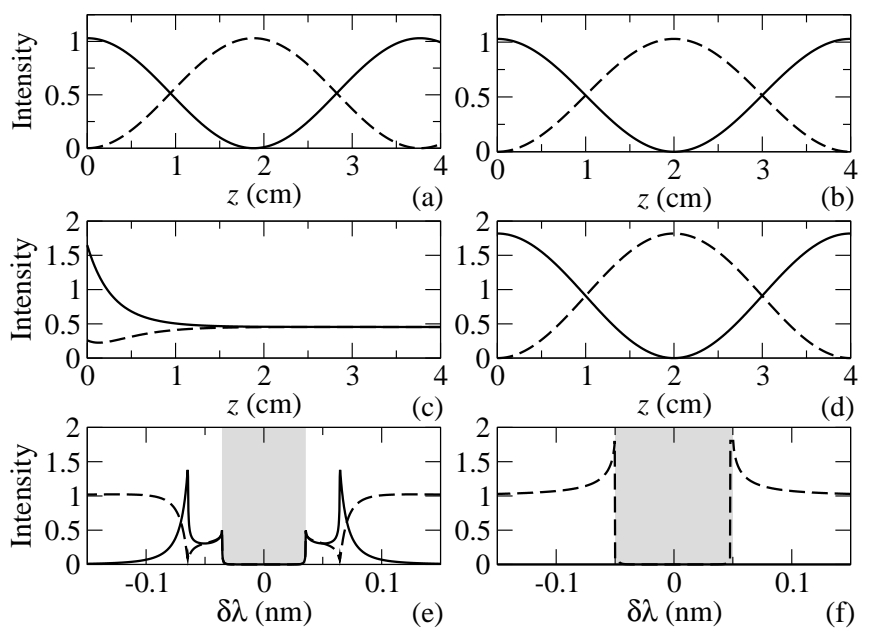

Fig. 2. Linear transmission of incident wave coupled to the first waveguide of a semi-infinite $(z \geq 0)$ coupler with (a,c,e) in-phase and (b,d,f) out-of-phase Bragg gratings: (a-d) Intensity distribution (averaged over grating period) shown in the first (solid line) and second (dashed line) waveguides for $(a, b)$ large frequency detuning from the resonance and $(\mathrm{c}, \mathrm{d})$ frequency tuned close to the band edge with slow group velocity $v_{g}=0.1$. (e,f) Intensities at $z=2 \mathrm{~cm}$ vs. wavelength detuning. Parameters correspond to Fig. 1, and the intensities are normalized to the input intensity.

After substituting these expressions into the linearized coupler equations (with $\gamma=0$ ), we obtain the dispersion relation $\omega^{2}(\beta)=\beta^{2}+C^{2}+|\rho|^{2} \pm 2 C\left[\beta^{2}+\right.$ $\left.|\rho|^{2} \cos ^{2}(\varphi / 2)\right]^{1 / 2}$.

Slow-light propagation can be observed due to the reduction of the normalized group velocity $\left(v_{g}=d \omega / d \beta\right)$ when the pulse frequency is tuned close to the bandgap edge, where the propagating waves with real $\beta$ are absent. We find that different regimes of slow light can be realized depending on the structural parameters. (i) If $|\rho \cos (\varphi / 2) / C|>1$, the bandgap appears for $\omega^{2}<\omega_{g}^{2}=$ $C^{2}+|\rho|^{2}-2 C|\rho \cos (\varphi / 2)|$, and only a single forward propagating mode (with $v_{g}>0$ ) exists for the frequencies near the gap edges, see examples in Figs. 1( c,e). (ii) If $|\rho \cos (\varphi / 2) / C|<1$, the bandgap appears for $|\omega|<\omega_{g}=|\rho \sin (\varphi / 2)|$, and two types of the forward propagating modes (with $v_{g}>0$ ) exist simultaneously (in the regions with $\beta>0$ and $\beta<0$ ) for the frequencies arbitrarily close to the gap edges, see examples in Figs. 1 1 d,f).

We now analyze linear propagation of pulses in a semiinfinite Bragg grating coupler. When the optical frequency is detuned from the bandgap, light periodically tunnels between the waveguides with the characteristic period $L_{c} \simeq \pi /(2 C)$ defined for a conventional coupler without the Bragg grating, see examples in Figs. 2( $(a, b)$. The periodic tunneling appears due to the beating of even and odd modes, which correspond to different branches

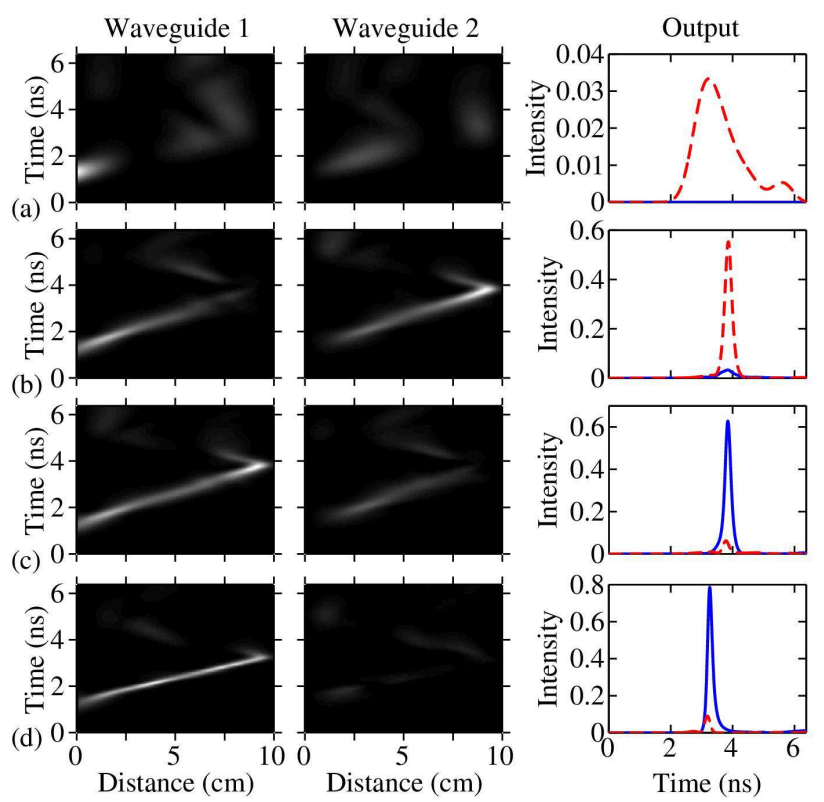

Fig. 3. (a-d) Pulse dynamics inside the nonlinear coupler for different values of the normalized peak input intensities $I_{0}=10^{-4}, 3.33,3.37,4$. Shown are the density plots of intensity in the first (left column) and second (middle column) waveguides. Output intensity profiles normalized to $I_{0}$ at the first (solid line) and second (dashed line) waveguides are shown in the right column. Input Gaussian pulse has full width at half-maximum of intensity of $577 p s$, and its central wavelength is tuned to the gap edge at $\lambda_{0}-\Delta \lambda_{0} / 2$.

of the dispersion curves. When the pulse frequency is tuned closer to the gap edge and (i) only one slow mode is supported, then periodic beating disappears and light is equally distributed between the waveguides irrespective of the input excitation, see Figs. 2( $(c, e)$. The periodic coupling can only be sustained in the slow-light regime when (ii) two modes co-exist at the gap edge, see Figs. 2(d,f). Therefore, the configuration with out-ofphase shifted Bragg gratings is the most preferential for switching of slow-light pulses, since for $\varphi=\pi$ the dispersion of the type (ii) is always realized for any values of the grating strength and the waveguide coupling, and simultaneously the bandgap attains the maximum bandwidth.

At higher optical powers, nonlinear effects become important, and we perform numerical simulations of the coupler equations to model pulse propagation. Examples of the pulse dynamics and output pulse characteristics are presented in Figs. 3 and 4 , where we consider the structure size equal to three coupling lengths, $L=3 L_{c}$. In the linear regime, the pulse tunnels three times between the waveguides and switches accordingly to the other waveguide at the output, see Fig. 3(a). However, at the same time the pulse significantly broadens due to the effect of the group-velocity dispersion (GVD). As 


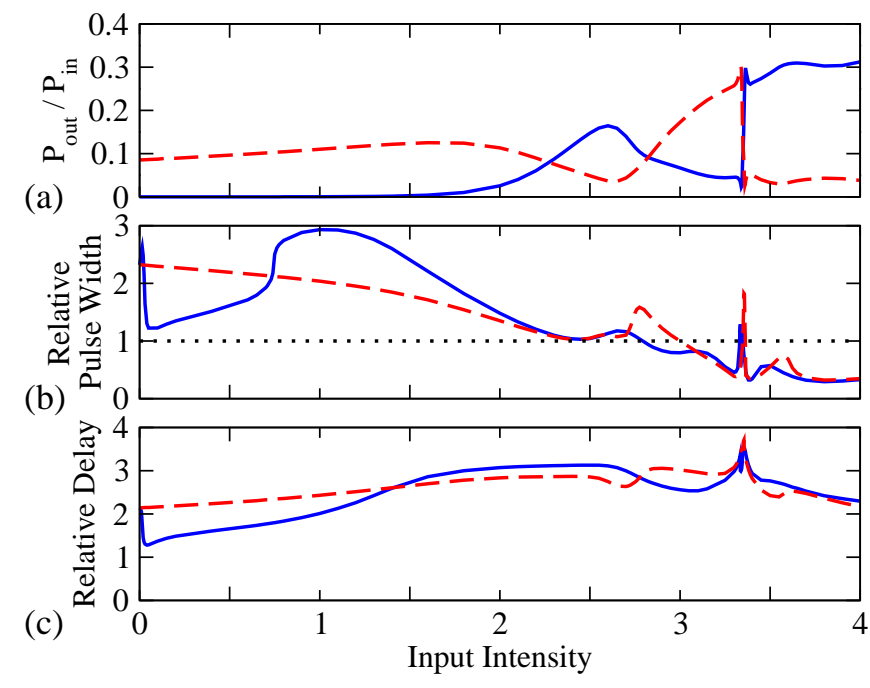

Fig. 4. Dependence of output pulse characteristics on the input peak intensity: (a) output power normalized to the input power; (b) pulse full-width at half-maximum of intensity, dotted line marks the input pulse width; (c) pulse delay relative to propagation without the Bragg grating normalized to the input pulse width. In all the plots, solid and dashed lines correspond to the outputs at the first and second waveguides, respectively.

the input pulse energy is increased, nonlinearity may support dispersionless slow-light pulses in the form of gap solitons, studied previously in single $\mathrm{e}^{5}$ and coupled waveguides with in-phase gratings $16,17,18$. Most remarkably, we find that the presence of two types of slowlight modes in the structure with out-of-phase gratings gives rise to a new type of gap solitons which periodically tunnel between the waveguides while preserving a constant width, see Figs. 3(b-d) and Fig. 4(b). In agreement with the properties of conventional nonlinear couplers $7,8,9$, the coupling length is gradually extended as the optical power is increased, resulting in the pulse switching between the output waveguides, see Fig. 4(a). As the input power is further increased, we observe a sharp switching when the output is highly sensitive to small changes of the input intensity (less than 1\%), cf. Figs. 3(b) and (c). At the same time, the pulse delay is also varied with optical power, as shown in Fig. 4(c). The power tunability of the pulse delay and switching dynamics can be adjusted by selecting parameters such as waveguide coupling, and choosing the frequency detuning from the gap edge.

In conclusion, we have demonstrated that flexible manipulation of slow-light pulses can be realized in a nonlinear couplers with phase-shifted Bragg gratings, implemented as all-fiber ${ }^{13}$ or planar waveguide devices created in highly nonlinear materials ${ }^{20,21}$. We predict the possibility to simultaneously suppress pulse spreading due to dispersion, all-optically tune the pulse velocity and transit delays, and switch pulses between the output ports.
We anticipate that similar effects may be achieved in other types of photonic structures including photoniccrystal waveguides $^{22}$ and fibers ${ }^{23}$ engineered to support several co-propagating slow-light modes. Our results also suggest new opportunities for control of slow-light bullets in periodic waveguide arrays 24 .

We thank M. de Sterke and B. Eggleton for useful discussions. This work has been supported by the Australian Research Council.

\section{References}

1. Y. A. Vlasov, M. O'Boyle, H. F. Hamann, and S. J. McNab, Nature 438, 65 (2005).

2. H. Gersen, T. J. Karle, R. J. P. Engelen, W. Bogaerts, J. P. Korterik, N. F. Hulst, van, T. F. Krauss, and L. Kuipers, Phys. Rev. Lett. 94, 073903 (2005).

3. R. S. Jacobsen, A. V. Lavrinenko, L. H. Frandsen, C. Peucheret, B. Zsigri, G. Moulin, J. Fage Pedersen, and P. I. Borel, Opt. Express 13, 7861 (2005).

4. M. Soljacic, S. G. Johnson, S. H. Fan, M. Ibanescu, E. Ippen, and J. D. Joannopoulos, J. Opt. Soc. Am. B 19, 2052 (2002).

5. J. T. Mok, C. M. de Sterke, I. C. M. Littler, and B. J. Eggleton, Nature Physics 2, 775 (2006).

6. R. J. P. Engelen, Y. Sugimoto, Y. Watanabe, J. P. Korterik, N. Ikeda, N. F. Hulst, van, K. Asakawa, and L. Kuipers, Opt. Express 14, 1658 (2006).

7. S. M. Jensen, IEEE Trans. Microw. Theory Tech. MTT-30, 1568 (1982).

8. A. A. Maier, Kvantov. Elektron. 9, 2296 (1982) (in Russian) [Quantum Electron. 12, 1490 (1982)].

9. S. R. Friberg, Y. Silberberg, M. K. Oliver, M. J. Andrejco, M. A. Saifi, and P. W. Smith, Appl. Phys. Lett. 51, 1135 (1987).

10. S. S. Orlov, A. Yariv, and S. Van Essen, Opt. Lett. 22, 688 (1997).

11. G. Perrone, M. Laurenzano, and I. Montrosset, J. Lightwave Technol. 19, 1943 (2001).

12. S. Tomljenovic Hanic and J. D. Love, J. Opt. Soc. Am. A 22, 1615 (2005).

13. M. Aslund, J. Canning, L. Poladian, C. M. de Sterke, and A. Judge, Appl. Optics 42, 6578 (2003).

14. J. M. Castro, D. F. Geraghty, S. Honkanen, C. M. Greiner, D. Iazikov, and T. W. Mossberg, Appl. Optics 45, 1236 (2006).

15. M. Imai and S. Sato, in Photonics Based on Wavelength Integration and Manipulation, Vol. 2 of IPAP Books, K. Tada, T. Suhara, K. Kikuchi, Y. Kokubun, K. Utaka, M. Asada, F. Koyama, and T. Arakawa, eds., (2005), pp. 293-302.

16. W. C. K. Mak, P. L. Chu, and B. A. Malomed, J. Opt. Soc. Am. B 15, 1685 (1998).

17. W. C. K. Mak, B. A. Malomed, and P. L. Chu, Phys. Rev. E 69, 066610 (2004).

18. A. Gubeskys and B. A. Malomed, Eur. Phys. J. D 28, 283 (2004). 
19. G. P. Agrawal, Nonlinear Fiber Optics (Academic Press, New York, 1988).

20. P. Millar, R. M. De la Rue, T. F. Krauss, J. S. Aitchison, N. G. R. Broderick, and D. J. Richardson, Opt. Lett. 24, 685 (1999).

21. M. Shokooh Saremi, V. G. Ta'eed, N. J. Baker, I. C. M. Littler, D. J. Moss, B. J. Eggleton, Y. L. Ruan, and B. Luther-Davies, J. Opt. Soc. Am. B
23, 1323 (2006).

22. D. Mori and T. Baba, Opt. Express 13, 9398 (2005).

23. M. Ibanescu, S. G. Johnson, D. Roundy, C. Luo, Y. Fink, and J. D. Joannopoulos, Phys. Rev. Lett. 92, 063903 (2004).

24. A. A. Sukhorukov and Yu. S. Kivshar, Phys. Rev. Lett. 97 (2006), in press. 